\title{
Effectiveness of Ciprofloxacin and Levofloxacin in treatment of Pseudomonas Aeruginosa infection
}

\author{
Imtiaz Ahmad', Wasil Khan², Aleem ur Rashid², Samiullah³ ${ }^{3}$ Abdul Ahad², Aminullah², S.M.Naeem ${ }^{4}$
}

\begin{abstract}
Background: Pseudomonas aeruginosa is the most troublesome and feared pathogen in medical field. It is the sixth most common cause of hospital-acquired infections and the cause of ventilator-associated pneumonia. The most common multidrugresistant gram-negative pathogen in VentilatedAssisted Patient. In our study in 100 patients culture and sensitivity report only $46 \%$ were sensitive to oral treatment while $54 \%$ of the pseudomonas were resistant to ciprofloxacin and $34 \%$ sensitive and $66 \%$ resistant to levofloxacin.

Objective: To find out resistance of pseudomonas to oral medication.

Material and Methods: A cross-sectional study, conducted in Saidu Group of Teaching Hospital from January 2018 to July 2019. 100 patient samples (tracheal aspirate, bronchial washing, sputum, pus, urine and blood) were cultured for pseudomonas and its sensitivity to oral medication ciprofloxacin and levofloxacin was recorded.

Results: Sensitivity to $46 \%$ were samples was observed to oral treatment while $54 \%$ of the pseudomonas were resistant to ciprofloxacin and $34 \%$ sensitive and $66 \%$ resistant to levofloxacin respectively.

Conclusion: It was found that $54 \%$ pseudomonas were resistant to Ciprofloxacin and $66 \%$ resistant to levofloxacin. So we should always consider for combination therapy.

Key words: Pseudomonas, oral medication, culture and sensitivity, resistance

This article may be cited as: Ahmad I, Khan W, Rashid UA, Samiullah, Ahad A, Aminullah, Naeem SM

Effectiveness of Ciprofloxacin and Levofloxacin in treatment Pseudomonas Aeruginosa infection. J Saidu Med Coll Swat 2020;10(1):79-82
\end{abstract}

\section{INTRODUCTION}

Pseudomonas is a rod-shaped, aerobic, Gramnegative bacterium belonging to the family Pseudomonadaceae ${ }^{1}$. Pseudomonas aeruginosa easily adapts to the environment it inhabits and can also colonize and invade a human host to cause serious infections ${ }^{2,3}$. P. aeruginosa isolates that cause infections are thought to express various virulence factors. This pathogen is one of the most common causes of pneumonia ${ }^{4,5}$.

Risk factors for the development of infections caused by Pseudomonas include neutropenia, cystic fibrosis, severe burns, and foreign device installations ${ }^{2,3}$. The general human population is refractory against infections caused by Pseudomonas species, but Pseudomonas species are physiologically highly flexible and able to act as opportunistic pathogens in humans with weakened immune systems ${ }^{6}$. P. aeruginosa causes life-threatening community-acquired pneumonia, nosocomial infections such as pneumonia, urinary tract infections, bacteremia and chronic lung infections in patients with cystic

\footnotetext{
1.Department of Pathology Saidu Medical College, Swat.

2.Department of Medicine Saidu Medical College, Swat.

3.Department of Nephrology, Saidu Medical College, Swat

4.Department of Biochemistry, Saidu Medical College, Swat.

Correspondence: Dr. Imtiaz Ahmad

Associate Professor of pathology, Saidu Medical College, Swat. Email: imtiaz.ahmad71@gmail.com

Cell: 0345-8880737
}

Received October 28, 2019 Accepted March 2, 2020 fibrosis $^{7}$. The identification of the microbiological cause of a case of pneumonia is especially important for preservation of the sensitivity of bacteria to antibiotics and for regulation of drug therapy $^{7}$.

One of the most important challenges for physicians is the adequate treatment of infections due to Gram-negative pathogens because of the increasing antimicrobial resistance in the healthcare setting ${ }^{8}$. Among infections caused by Gram-negative rods, Pseudomonas aeruginosa has a leading role ${ }^{9}$, especially in critically ill and immunocompromised patients. Antimicrobial resistance has led to a serious restriction in treatment options for $\mathrm{P}$. aeruginosa infections, which has become a critical and deadly issue causing a total of 51,000 healthcare infections in the USA per year ${ }^{10,11}$.

According to data reported to the National Healthcare Safety Network (NHSN) in the United States from 2011 to 2014 , P. aeruginosa is ${ }^{12}$. The sixth most common cause of hospital-acquired infections in general (7.3 percent) The second most common cause of ventilator-associated pneumonia (VAP; 16.5 percent) and the most common multidrug-resistant gram-negative pathogen causing VAP. The third most common cause of catheter-associated urinary tract infections (10.3 percent). The fifth most common cause of surgical site infections (5.7 percent).It is also a common pathogen worldwide; it was the 
third most common cause of intra-abdominal infections from 2002 to 2011 and the third most common cause of urinary tract infections from 2009 to $2011^{13}$.

\section{MATERIALAND METHODS}

This cross-sectional study was conducted on one hundred (100) patients' in Saidu Group of Teaching Hospital from January 2018 to July 2019. Demographic details including age, gender, location and clinical features were recorded. The isolate were collected from tracheal aspirate, bronchial washing, sputum, pus, urine and blood specimens of patients' of different units including intensive care unit, pediatric, medical and gynecology units.

Clinical isolate were identified by standard methods . they were inoculated on Blood agar (Oxide), Mac Conkeys agar (Oxide)and Cystine lactose electrolyte deficient agar (Oxide). Isolate were identified on the basis of colony morphology, Gram Staining and biochemical tests including Catalase, Oxidase, Sulfide, Indole, Motality, Citrate, urea, TSI reaction, Pyocinin production and lactose. Mueller Hinton plates were seeded with 0.5 MacFarland suspension matched turbidity inocula and antibiotic Disc were placed on them.

Results were interpreted after 24hours of incubation at $37^{\circ} \mathrm{C}$ by measuring zones of antibiotics around discs. Isolates that were not identified on the basis of conventional methods was carried out by analytical Profile Index (API) 20 NE kit (BioMerieux, France).

Antibiotic susceptibility test was done by using the disc diffusion Kirby Bauer method. The antibiotics tested include Levofloxacin , Ciprofloxacin Tazobactum/ Piperacillin 10/100Mcg, amikacin (30mcg) Gentamycin (10mcg), Ceftazidime $10 \mathrm{mcg}$, cefepime $(10 \mathrm{mcg})$, meropenem $(10 \mathrm{mcg})$, imipenem (10mcg), cefoferazone/sulbactam and Colistin. Ps. Aeruginosa ATCC-27853 was used as reference strain.

\section{RESULTS}

In 100 patients' culture $46 \%$ were sensitive to ciprofloxacin and $54 \%$ were resistant while there is $34 \%$ sensitivity and $66 \%$ resistance to levofloxacin.

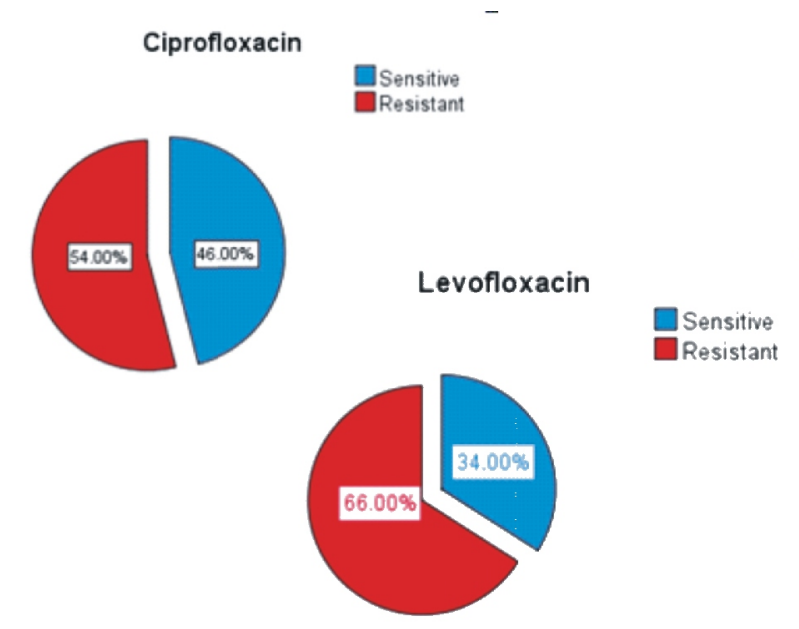

\section{Statistics}

\begin{tabular}{|c|c|c|c|}
\hline & & Ciprofloxacin & Levofloxacin \\
\hline \multirow[t]{2}{*}{$\mathrm{N}$} & Valid & 100 & 100 \\
\hline & Missing & 0 & 0 \\
\hline
\end{tabular}

\begin{tabular}{|c|c|c|c|c|c|}
\hline \multicolumn{6}{|c|}{ Ciprofloxacin } \\
\hline & & Frequency & Percent & Valid Percent & $\begin{array}{c}\text { Cumulative } \\
\text { Percent }\end{array}$ \\
\hline \multirow[t]{3}{*}{ Valid } & Sensitive & 46 & 46.0 & 46.0 & 46.0 \\
\hline & Resistant & 54 & 54.0 & 54.0 & 100.0 \\
\hline & Total & 100 & 100.0 & 100.0 & \\
\hline \multicolumn{6}{|c|}{ Levofloxacin } \\
\hline & & Frequency & Percent & Valid Percent & $\begin{array}{c}\text { Cumulative } \\
\text { Percent }\end{array}$ \\
\hline \multirow[t]{3}{*}{ Valid } & Sensitive & 34 & 34.0 & 34.0 & 34.0 \\
\hline & Resistant & 66 & 66.0 & 66.0 & 100.0 \\
\hline & Total & 100 & 100.0 & 100.0 & \\
\hline
\end{tabular}




\section{DISCUSSION}

Pseudonas aeruginosa is often an opportunistic pathogen, causing infections in patients with physical, phagocytic, or immunologic defects in host defense mechanisms. Historically, P. aeruginosa has been a major burn wound pathogen, a serious cause of bacteremia in neutropenic patients, and the most important pathogen in cystic fibrosis (CF) patients. However, these associations have undergone considerable change, with a shift in the spectrum of the hosts who are now commonly infected by $P$. aeruginosa.

In our study in 100 patients culture and sensitivity report only $46 \%$ were sensitive to oral treatment while $54 \%$ of the pseudomonas were resistant to ciprofloxacin and $34 \%$ sensitive and $66 \%$ resistant to levofloxacin. In Pakistan there is increase resistance to antibiotic because of irrational and over the counter use.

Better ways of administering antibiotics have been considered to overcome the increasing resistance patterns. In a pharmacodynamic analysis, highdose, prolonged infusions of cefepime, ceftazidime, doripenem, and meropenem had the highest probabilities of achieving bactericidal exposure for P. aeruginosa ${ }^{14}$.

Acquired resistance, specifically, can result from mutation or acquisition of exogenous resistance determinants and can be mediated by a number of mechanisms, including degrading enzymes, reduced permeability, and active efflux ${ }^{15,16}$.

Resistance to carbapenems, particularly imipenem, can arise by simple mutation resulting in the loss of a carbapenem-specific porin, $O p r D^{17}$.

Syed Maisam Ali etal have shown 39\% resistance to ciprofloxacin ${ }^{18}$. Astudy conducted in In Turkey in 1996, revealed only $94 \%$ sensitivity of Pseudomonas aeruginosa to ciprofloxacin, ${ }^{19}$ while the resitance went higher to $100 \%$ of the isolates, showed resistance to ciprofloxacin ${ }^{20}$.

Patterns of antibiotic resistance in P. aeruginosa evolve over time and vary geographically and by type of infection. This geographical variation highlights the importance of local epidemiological data when choosing empiric therapy.

As an example, in North America from 2005 to 2010 , resistance to fluoroquinolones among intra- abdominal and urinary tract infection isolates increased (from 22 to 33 percent), resistance to imipenem (20 percent) and other beta-lactams ( $p$ ip er acil lin-ta zo ba ct am, ce fe pime, an d ceftazidime; 23 to 26 percent) remained essentially unchanged, and resistance to amikacin declined (from 11 to 3 percent) ? Resistance patterns were different in other locations: in South Africa, resistance to piperacillin-tazobactam was 8 percent and to cefepime, ceftazidime, and imipenem was 25 to 26 percent; in China, resistance rates to amikacin and piperacillin-tazobactam were 12 and 8 percent, respectively.

In the United States specifically, surveillance suggests a trend towards declining rates of resistance, although rates still remain high ${ }^{6}$. Among catheter-related bloodstream infection, catheter-associated urinary tract infection, and ventilator associated pneumonia isolates, resistance ranged from 22 to 26 percent for extended-spectrum cephalosporins, 16 to 19 for piperacillin-tazobactam, 30 to 33 percent for fluoroquinolones, 24 to 28 percent for carbapenems, and 17 to 23 percent for aminoglycosides. Approximately 18 to 19 percent were multidrug-resistant. Rates of resistance were generally lower (4 to 11 percent) among surgical site infection isolates.

\section{CONCLUSION}

Pseudomos Aeruginosa resistance is increasing day by day through different mechanism especially to oral antibiotic i.e. ciprofloxacin and levofloxacin.

RECOMMENDATIONS: Irrational use of antibiotic needs to be stopped. Over the counter antibiotic should be banned and should be only given according to Culture \& Sensitivity. There should be check and balance of antibiotic use in veterinary and poultry industry by concern authorities as they are giving antibiotic and that also contributes in the development of resistance.

Further research should be made to establish the fact and develop new antibiotics, and combination of Intravenous antibiotics should be used for treatment and prevention of infection. 


\section{REFERENCES}

1. Bentzmann S, Plésiat $P$. The Pseudomonas aeruginosa opportunistic pathogen and human infections. Environ. Microbiol. 2011; 13: 1655-1665.

2. Hardalo C, Edberg SC. Pseudomonas aeruginosa: assessment of risk from drinking water. Crit. Rev. Microbiol. 1997; 23: 47-75..

3. Mena KD, Gerba CP. Risk assessment of Pseudomonas aeruginosa in water. Rev Environ Contam. Toxicol. 2009201, 711-15..

4. Zarb P, Coignard B, Griskeviciene J, Muller A, Vankerckhoven V, Weist K, et al. The European Centre for Disease Prevention and Control (ECDC) pilot point prevalence survey of healthcare-associated infections and antimicrobial use. Euro Surveill. 2012; 17: 203-16.

5. Kollef $\mathrm{MH}$, Chastre J, Fagon JY, François $\mathrm{B}$, Niederman MS, Rello J, et al. Global prospective epidemiologic and surveillance study of ventilatorassociated pneumonia due to Pseudomonas aeruginosa. Crit. Care Med. 2014; 42:2178-87.

6. Bubonja-Sonje M, Matovina M, Skrobonja I, Bedenic B, Abram M. Mechanisms of Carbapenem Resistance in Multidrug-Resistant Clinical Isolates of Pseudomonas aeruginosa from a Croatian Hospital. Microb Drug Resist. 2015;

7. Tran CS, Rangel SM, Almblad H, KierbelA, Givskov M, Tolker-Nielsen T, et al. The Pseudomonas aeruginosa type III translocon is required for biofilm formation at the epithelial barrier. PLoS Pathog. 2014; 10: 1004479.

8. Pena C, Suarez C, Tubau F, Dominguez A, Sora M, Pujol M, Gudiol F, Ariza J. Carbapenem-resistant Pseudomonas aeruginosa: factors influencing multidrug-resistant acquisition in non-critically ill patients. Eur J Clin Microbiol Infect Dis. 2009;28(5):519-22.

9. El Zowalaty ME, AI ThaniAA, Webster TJ, El Zowalaty AE, Schweizer HP, Nasrallah GK, Marei HE, Ashour HM. Pseudomonas aeruginosa: arsenal of resistance mechanisms, decades of changing resistance profiles, and future antimicrobial therapies. Future Microbiol. 2015;10(10):168370-6.

10. Fujii A, Seki M, Higashiguchi M, Tachibana I, Kumanogoh A, Tomono K. Community-acquired, hospital-acquired, and healthcare-associated pneumonia caused by Pseudomonas aeruginosa. Respir Med Case Rep. 2014;12:303.
11. Lund-Palau $H$, Turnbull AR, Bush $A$, Bardin $E$, Cameron L, Soren O, Wierre-Gore N, Alton EW, Bundy JG, Connett G Faust SN, Filloux A, Freemont P, Jones A, Khoo V, Morales S, Murphy R, Pabary R, Simbo A, Schelenz S, Takats Z, Webb J, Williams HD, Davies JC. Pseudomonas aeruginosa infection in cystic fibrosis: pathophysiological mechanisms and therapeutic approaches. Expert Rev Respir Med. 2016;10(6):685-97.

12. Weiner LM, Webb AK, Limbago B, et al. AntimicrobialResistant Pathogens Associated With HealthcareAssociated Infections: Summary of Data Reported to the National Healthcare Safety Network at the Centers for Disease Control and Prevention, 2011-2014. Infect Control Hosp Epidemiol 2016; 37:1288.

13. Morrissey I, Hackel M, Badal R, . A Review ofTen Years of the Study for Monitoring Antimicrobial Resistance Trends (SMART) from 2002 to 2011. Pharmaceuticals (Basel) 2013; 6:133-5.

14. Koomanachai P, Bulik CC, Kuti JL, Nicolau DP. Pharmacodynamic modeling of intravenous antibiotics against gram-negative bacteria collected in the United States. Clin Ther 2010; 32:766.

15. Livermore DM. Interplay of impermeability and chromosomal beta-lactamase activity in imipenemresistant Pseudomonas aeruginosa. Antimicrob Agents Chemother 1992; 36:2046.

16. Yokoyama K, Doi Y, Yamane K, et al.Acquisition of $16 \mathrm{~S}$ rRNA methylase gene in Pseudomonas aeruginosa. Lancet 2003; 362:1888.

17. Masuda N, Gotoh N, Ishii C. Interplay between chromosomal beta-lactamase and the MexAB-OprM efflux system in intrinsic resistance to beta-lactams in Pseudomonas aeruginosa. Antimicrob Agents Chemother 1999; 43:400.

18. Ali MS, Mahesar HJ, Shezad J, Zaman A, Shinwari W, Sajid T, Khatak KS. Spectrum of Pseudomonas aeruginosa sensitivity in chronic otitis media. J Saidu Med Coll Swat 2019;9(2): 191-4.

19. Altuntas A, Aslam A, Eren A, Unal A, Nalca Y. Susceptibility of microorganisms isolated from chronic te a c h in g h o s p i t a I to ciprofloxacin. Eur Arch Otorinolyringol. 1996;253:364-6.

20. Jang $\mathrm{CH}$, Park SY. Emergence of ciprofloxacin resistant pseudomonas in chronic suppurative otitis media. Cli Otolaryngol. 2004;29:321-3 